\title{
Real-Time 3D Motion Recognition of Skeleton Animation Data Stream
}

\author{
Jianchao Lv and Shuangjiu Xiao
}

\begin{abstract}
In this paper, a method for real-time 3D motion recognition based on a hierarchical recognition framework is presented. To facilitate the recognition process, motions are divided into three levels by duration and complexity. SVD (Singular Value Decomposition) is used to extract the feature vector of each motion matrix, and SVM(Support Vector Machine) is utilized to do the training and classification of the first level of motion(sub-motion). In motion recognition process, the sequence of recognized candidate sub-motions is analyzed by HMM (Hidden Markov Model) to gain certain robustness, then we recognize the second level of motions by pattern matching in this sequence. Finally a grammar-based motion synthesization is applied using motions as semantic terms to recognize the third level of motions. Experimental results show that the proposed method has high performance in sensitivity, accuracy, specialty and efficiency.
\end{abstract}

Index Terms-Motion recognition, support vector machine, singular value decomposition, grammar, classification.

\section{INTRODUCTION}

Recently, motion recognition can find wide applications in virtual reality as it provides a more nature way of humanmachine interaction. It depends on MoCap (motion capture system) as its data provider, inertial-based MoCap has caught more and more attentions due to its capability of providing reliable real-time motion data in an explicit way [1]. Data streams generated from these MoCaps have multiple attributes, each of which is the kinematics parameter of one sampling point or joint of a human skeleton [2]. The multi-attribute property poses certain challenges to real-time motion recognition.

- Motions differ in duration and complexity: they can be short and simple, they can be long and sophisticated, they should be handled differently.

- Massive data to deal with: real-time motion streams have thousands of rows of multi-attribute data, which need to be reduced to data of small scales while still representative of previous data.

- Spatio-temporal variations of similar motions: as they may be of different durations, trajectories and scales, etc. Similar motions doesn't necessarily result in similar data, the effect of variations need to be eliminated.

Motion recognition algorithms can be roughly grouped into four classes. First, the most intuitive way is template matching, usually there is a distance metric to make pair wise comparison. Dynamic programming and DTW (dynamic time warping) and their variations are often used

Manuscript received July 1, 2013; revised Septemeber 5, 2013.

The authors are with Shanghai Jiaotong University/School of Software, Shanghai, P. R. China (e-mail: 1vjianchao@sjtu.edu.cn, xsjiu99@cs.sjtu.edu.cn). in similarity comparison. Ref. [3] created a citation structure called match web by using DTW, the structure is used for recognize similar motions. Ref [4] used CDP (Continuous Dynamic Planning) to locate and recognize gestures from continuous gesture sequences. Although DTW can take care of the time sequence alignment, its recognition effect is still affected by the spacial variations of two similar motions. Second, methods based on classification bring machine learning algorithms to this field. Michalis Taptis applies logistic regression to evaluate how well the dancer's performance matches with the canonical model [5] and obtains good results, however, motion curves, which are its proposed features, are not good abstraction but rather another way of presentation of motion data. Third, methods based on statistical model include HMM [6]-[8], DBN (Dynamic Bayes Network) [9], etc. Well trained statistic model can unveil the implicit statistical relations in gestures or motion sequences, thus help eliminate spatio-temporal variations in recognition process. Finally, methods based on grammar are used for good recognition of sophisticated, enduring and usually interactively motions, Ref. [10] proposed a context-free grammar to recognize motion in complicated scenarios. Methods based on grammar depend on the recognition of lower level of motions, therefore, importance lies very much in the lower level of motion recognition.

In this paper, we propose a motion recognition method based on a hierarchical recognition framework as we divide motions into three levels by their durations and complexities. The system consists of an offline training module and a realtime recognition module. We recorded thousands of submotions (300 for each) for training, the source data is cut by a shifting window to form the sub-motion matrices, and then SVD is conducted on each matrix to extract feature vector. We utilize SVM to train the labeled feature vectors of every two motion classes, in recognition, the trained models are used to make prediction of the most likely sub-motion. The sub-motion sequence is filtered by HMM and then assembled to be recognized as motions, the motions are basic tokens of our pre-defined grammars to synthesize motions into sentences as high level of messages or controlling commands in human-machine interaction.

Key contributions of the proposed method include:

- A hierarchical frame work: divide motion into three different levels and a framework consists of motion training and recognition pipelines to provide appropriate methods for each level of motion.

- Motion data abstraction and spatio-temporal variation elimination: using SVD, one-versus-one multi-class SVM and HMM.

- Definition of an extendable grammar: allow the 
machine understand higher levels of human motions.

The remainder of this paper is organized as follows. Section 2 describes the proposed method in details. Section 3 shows the results of our method and in Section 4, future works are discussed and conclusions are drawn.

\section{OUR APPROACH}

\section{A. Motion Data Source and Skeleton Representation}

Vision-based motion capture systems often encounter problems such as occlusion of parts of the body, background noise, restrictions such as lighting or speed of movement. Moreover, in order to capture a 3D motion, multiple cameras need to set up, thus the user's moving is restricted in the cameras view.All of these disadvantages of visionbased motion capture system may hinder the motion recognition's efficiency, sensitivity and accuracy, to overcome this, we use the motion capture system based on inertial sensing.It can tracking a user's subtle motion with fast speed,high precision in a direct way, all need to do is putting on a suit with certain number of inertial sensors, each sensor is called a IMU (Inertial Measurement Unit), itcan detect current rate of acceleration with accelerators, detect changes in pitch, roll and yaw bygyroscopes and calibrate against orientation drift using magnetometers.In our experiment, we use Animazoo IGS180-I suit, of whichthere are 18 IMUs mountedin it (see Fig. 1). Each IMUcan provide this body part's real-time rotational data in quaternions(at the rate of 50fps), all of them are connected by daisy chain wire, thus an animation skeletonis formedwith head as its referenceroot(see Fig. 1), as head skin has leastsoft tissues which can affect the IMU's alignment.

\section{B. Motion Levels}

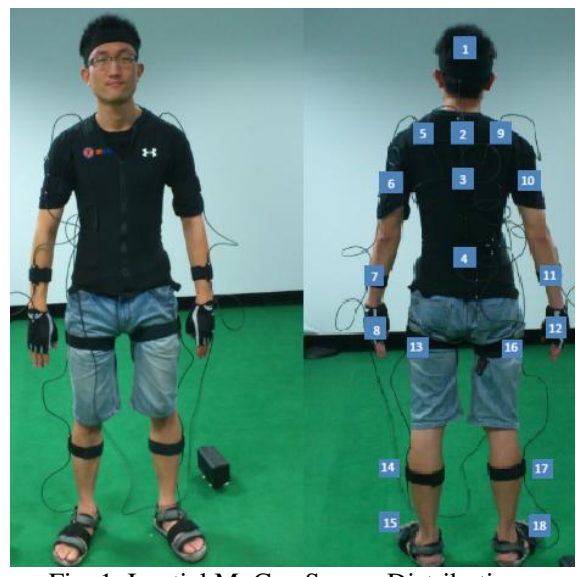

Fig. 1. Inertial MoCap Sensor Distribution.

Motions vary in durations and complexities, Aaron F. Bobick attaches importance to dividing motions into different levels in perception of motions, the division, as he argues, is necessary in making explicit both there presentational competencies and manipulations in motion recognition[11]. Most motion recognition methods focus on one level of motion or even mix up with different levels, nevertheless, the higher level of motion is based on the lower one and they should be treated with different methods, after all, there isn't a method applicable everywhere. In this paper, we divide motions into three levels:

- $\mathbf{L}_{\mathbf{1}} \mathbf{M}$ : the atomic motion (or movement), which is considered the unit of recognition by our approach, and is the sub-motion of $\mathrm{L}_{2} \mathrm{M}$.

- $\quad \mathbf{L}_{\mathbf{2}} \mathbf{M}$ : the lexical motion (or activity), which is a motion that has lexical meaning and can act as the term of our grammar, for instance, the parameter motion, "Left Direction", which consists of a "lift up left hand over head" and two "leftward swing of left arm".

- $\mathbf{L}_{3} \mathbf{M}$ : the syntax meaningful motion is a sentence with which the computer can take as the messages or controlling commands to follow in virtual reality, which offers more natural and easy way of human machine interaction.

\section{Method Overview}

With overcoming the aforementioned difficulties of motion recognition in mind, a hierarchical framework of a training pipeline and a recognition pipeline is proposed in order to obtain high sensitivity and accuracy in recognition of all three levels of motions. In both training and recognition pipelines, the motion matrices are generated first. MoCap provides data at the rate of $50 \mathrm{fps}$, and we define a standard $\mathrm{L}_{2} \mathrm{M}$ 's duration is of 1 second, so there are 50 frames, each frame includes 18 quaternions. Therefore, motion matrices are $\times(s \cdot q)$ matrices where $n(50)$ denotes the number of frames, $s(18)$ the number of sensors and $q(4)$ the number of elements in a quaternion.

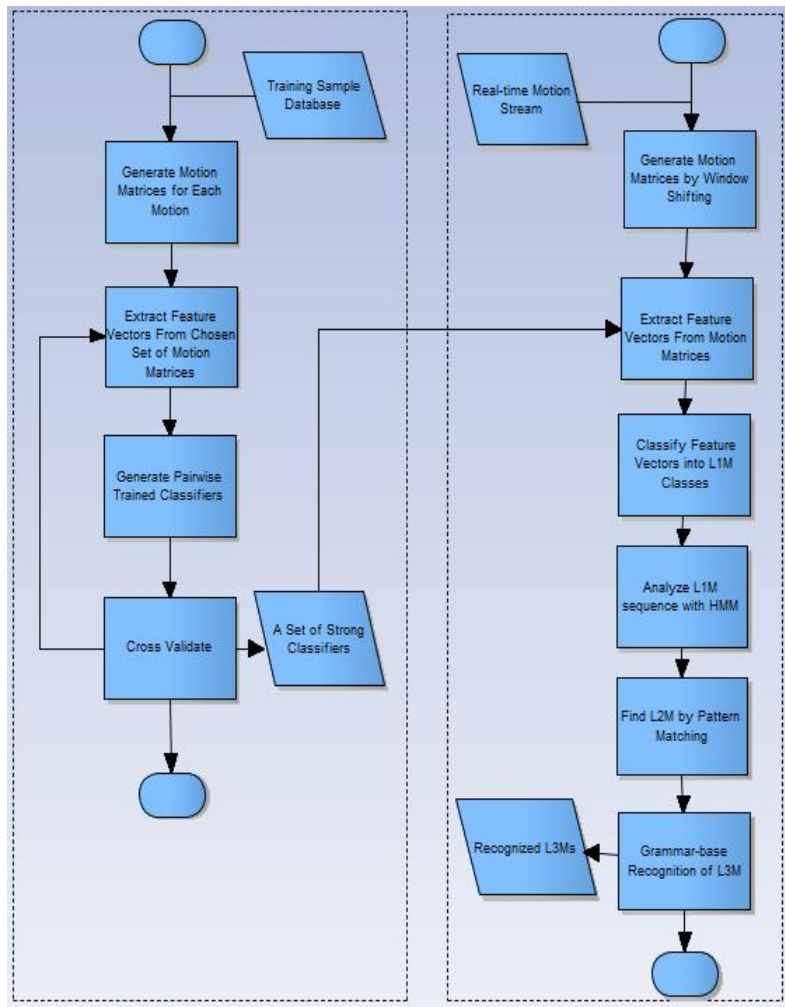

Fig. 2. The flowchart of motion training and recognition pipelines.

Feature vectors of a set of chosen motion matrices $(>300)$ are extracted by using SVD, and we propose combining the first and second singular vectors of motion matrices to form the feature vectors, which reveal the geometry structures of them. By training these feature vectors, we get a set of pairwise classifiers, the strong classifiers is finally chosen after cross validation to assure its quality, if accuracy of 
classification by cross validation is not ideal, we iterate this procedure as Fig. 2 illustrates. Finally, a set of $n(n-1) /$ 2 strong classifiers is settled, where $n$ denotes the number of $L_{1} \mathrm{Ms}$ and will be used in the recognition pipeline.

\section{Feature Extraction}

Motion matrices contain too much redundant information for classifiers, which will seriously affect the classification accuracy if applied SVM directly. Fig. 3 shows a 3D view of a motion matrix, although it has too many elements, its structural feature can be easily seen, an intuitive thinking is extracting the geometry structure from it. SVD is a basic while powerful tool to compute the geometry objects of a large matrix by decomposing it into three matrices, where each of them represent its information in a particular aspect. The decomposition depend on the theorem[12]: Let $A \in R^{m \times n}$ (real $m \times n$ matrix), then the following equation will be established:

$$
\mathrm{A}=\mathrm{U} \Sigma \mathrm{V}^{\mathrm{T}}
$$

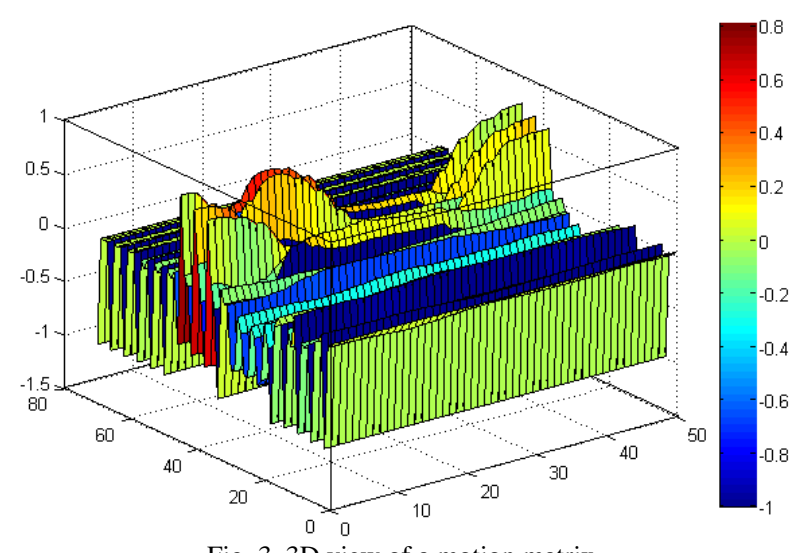

Fig. 3. 3D view of a motion matrix.

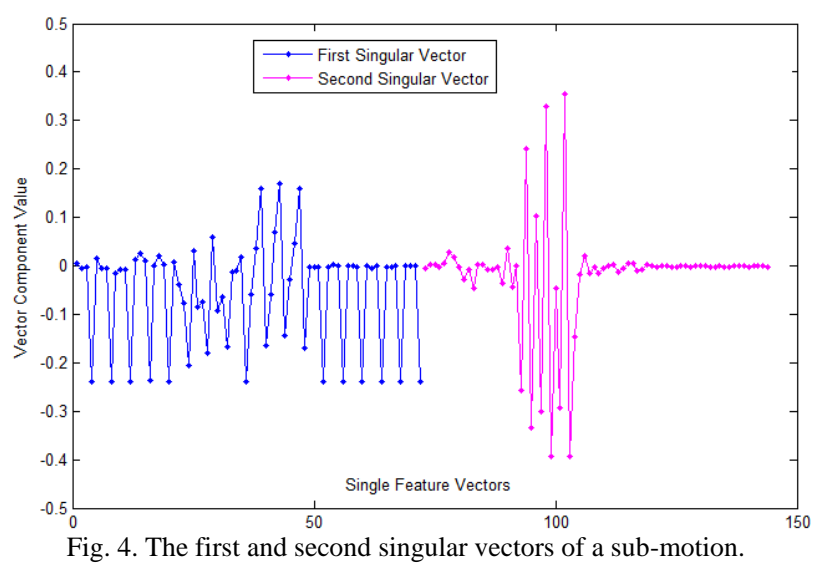

where $U \in R^{m \times m}$ (real $m \times n$ matrix) and $V \in R^{n \times n}$ (real $n \times n$ matrix) are orthogonal and unitary, and

$$
\Sigma=\left(\begin{array}{ll}
S & 0 \\
0 & 0
\end{array}\right)
$$

where $S=\operatorname{diag}\left(\delta_{1}, \delta_{2}, \ldots, \delta_{r}\right), r=\min (m, n)$, each collumn of matrix Uis a orthonormalized eigenvectors of $\mathrm{AA}^{\mathrm{T}}$ andits counterpart in $\mathrm{V}$ the orthogonal eigenvectors of $\mathrm{A}^{\mathrm{T}} \mathrm{A}$, and they are called left singular vector and right singular vector respectively, $\delta_{i}(i=1,2, \ldots, r)$ is called the singular value and is the nonegative square root of eigenvalues of $\mathrm{A}^{\mathrm{T}} \mathrm{A}$ and $\delta_{1} \geq \delta_{1} \geq \cdots \geq \delta_{r} \geq 0$. The right singular vectors well reveal the geometry structure of the matrix A, but differ in importance according to the singular values' size, our experiments show that only the first and second singular vector cannot be neglected when constructing the feature vectors. Fig. 4 illustratesthe first and second singular vectors of onemotion matrix ofa motion classwhile Fig. 5 shows first and second singular vectors of all motion matrices of the same motion class $(>300)$.One can easily tell two facts from it:

- Both the first and second singular vectors are insensitive to the motion variations among the same motion class as they fit well with each other.

- The first singular vectors are more insensitive as they vary less than the second singular vectors.

Feature vectors are constructed by concatenating the first and second singular vectors with different weights according to their related singular values.

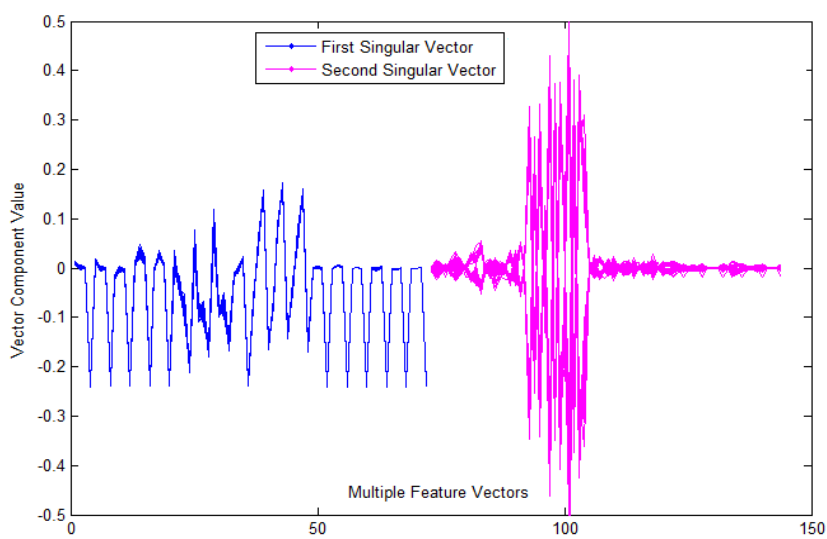

Fig. 5. The first and second singular vectors of all sub-motion in the same sub-motion class

\section{E. $L_{1}$ MRecognition by SVM and HMM}

SVM has demonstrated many successful applications in pattern recognition problems, which is widely acknowledged the best "off-the-shelf" supervised learning algorithm, where the concept of optimal margin classifier is introduced. Its key idea is finding the optimal hyper plane which maximizes the geometric margin as a decision boundary between two classes of training samples. The geometric margin of the decision boundary with respect to training sample $\left(x^{(i)}, y^{(i)}\right)$ can be defined as:

$$
\gamma^{i}=y^{(i)}\left(\left(\frac{\omega}{\|\omega\|}\right)^{T} x^{(i)}+\frac{b}{\|\omega\|}\right)
$$

where $(\omega, b)$ is the hyperplane, and all the points on it satisfy the equation $\omega^{T} x+b=0\left(\omega=\left[\theta_{1} \ldots \theta_{n}\right]^{T}\right.$ and $b=\theta_{0}$, they are the parameters to be calculated). The problem of maximize the geometric marginis equivalent to the following optimization problem:

$$
\min _{\gamma, \omega, b} \frac{1}{2}\|\omega\|^{2}
$$

$$
\text { s.t. } y^{(i)}\left(\omega^{T} x^{i}+b\right) \geq 1, i=1, \ldots, m
$$

The above problem's dual form when introducing Lagrange duality will allow us to use kernels to make the 
multi-class classifiable in very high dimensional spaces.

In recognition of $\mathrm{L}_{1} \mathrm{M}$, a one-versus-one multi-class SVM is used to decide which class of the sub-motion belongs to, each classifier is trained using the two classes involved, another method used in multi-class classification with binary classifiers is one-versus-rest, where one class is the positive sample and all the rest are negative samples. As is shown in [13], the former one outperforms the latter one in both accuracy and training time.

Hidden Markov Model is a statistic model which can be built upon a set of unobserved (hidden) states, while the states transitions are related to a Markov process. We use HMM to compute the latent $\mathrm{L}_{1} \mathrm{M}$, which is the hidden state, given the trained parameters of a learning algorithm and a sequence of previous recognized $\mathrm{L}_{1} \mathrm{Ms}$ as observations. The set of hidden states in our case is defined as:

$$
S=\left\{\text { state } \mid \text { state } \in L_{1} \mathrm{M}^{(i)}, \mathrm{i}=1, \ldots, n\right\}
$$

where $n$ is the number of our $\mathrm{L}_{1} \mathrm{Ms}$, and $\mathrm{L}_{1} \mathrm{M}^{(i)}$ is the ith one of all $\mathrm{L}_{1} \mathrm{Ms}$. The set of observation states, in our case, is the same set as $\mathrm{S}$, which is denoted by $\mathrm{O}$. We use a modified version of Viterbi algorithm to implement the HMM and it works well to find the most likely hidden $\mathrm{L}_{1} \mathrm{Ms}$ sequence given the observation of the candidate $\mathrm{L}_{1} \mathrm{M}$ sequences. The parameters of HMM is trained using Baum-Welch learning algorithm with our real-time recorded training samples.

\section{F. $L_{2} M$ Recognition by Pattern Matching}

One major problem in motion recognition is that logically similar motions are not necessarily numerically similar, there must be spatio-temporal variations between two similar motions executed differently while there also certain aspects of the similar motions which are consistent [14]. Fortunately, we have already recognized a number of $\mathrm{L}_{1} \mathrm{Ms}$, and they are the logically essence of $\mathrm{L}_{2} \mathrm{Ms}$, all we need to do is evaluate the sequence of current recognized $\mathrm{L}_{1} \mathrm{Ms}$ and decide which class of $\mathrm{L}_{2} \mathrm{M}$ are they in. Table I shows all defined motion patterns.

TABLE I: MOTION PATTERNS OF $\mathrm{L}_{2} \mathrm{M}$

\begin{tabular}{|c|c|c|c|}
\hline \multicolumn{4}{|c|}{ L1Ms involved and their orders } \\
\hline $\mathrm{Me}$ & Lift left hand & Pat chest & Pat chest \\
\hline Claps & Clap & Clap & Clap \\
\hline $\begin{array}{l}\text { Wear a } \\
\text { Scarf }\end{array}$ & Lift left hand & $\begin{array}{c}\text { Put left arm } \\
\text { around neck and } \\
\text { back }\end{array}$ & $\begin{array}{c}\text { Put left arm } \\
\text { around neck and } \\
\text { back }\end{array}$ \\
\hline See & Lift left hand & $\begin{array}{c}\text { Draw a circle } \\
\text { before eyes using } \\
\text { left hand }\end{array}$ & $\begin{array}{c}\text { Draw a circle } \\
\text { before eyes using } \\
\text { left hand }\end{array}$ \\
\hline Stop & $\begin{array}{l}\text { Stretch out left } \\
\text { arm to the left }\end{array}$ & Palm faces left & N/A \\
\hline $\begin{array}{l}\text { Stretch } \\
\text { Oneself }\end{array}$ & $\begin{array}{l}\text { Lift both left and } \\
\text { right arms to waist }\end{array}$ & $\begin{array}{l}\text { Stretch out both } \\
\text { arms over head }\end{array}$ & N/A \\
\hline $\begin{array}{c}\text { Left } \\
\text { Direction }\end{array}$ & $\begin{array}{l}\text { Lift left hand } \\
\text { overhead }\end{array}$ & $\begin{array}{l}\text { Swing left ward } \\
\text { and back }\end{array}$ & $\begin{array}{l}\text { Swing left ward } \\
\text { and back }\end{array}$ \\
\hline $\begin{array}{c}\text { Right } \\
\text { Direction }\end{array}$ & $\begin{array}{l}\text { Lift right hand } \\
\text { over head }\end{array}$ & $\begin{array}{l}\text { Swing right ward } \\
\text { and back" }\end{array}$ & $\begin{array}{l}\text { Swing rightward } \\
\text { and back }\end{array}$ \\
\hline
\end{tabular}

The motion pattern is easily adapted and extended when new motion classes are added.

\section{G. $L_{3} M$ Recognition by Grammar-Based Command Synthesization}

In the synthesization of $\mathrm{L}_{3} \mathrm{Ms}$ we introduce the notation for describing the production rules of the motions' grammar, from now, we will use $\mathrm{M}$ to denote the generic term of motion, S subject, VI intransitive verb, VT transitive verb, A adverbial adjunct and $\mathrm{O}$ object. And we also relate each $\mathrm{L}_{2} \mathrm{M}$ to one of the above grammatical roles, for instance, the $\mathrm{L}_{2} \mathrm{M}(\mathrm{Me})$ refers to $\mathrm{S}$ as subject grammatically, $\mathrm{L}_{2} \mathrm{M}$ (Wear a scarf) refers to VI as intransitive verb. The table below is the production rules of our defined grammar.

Together with speech recognized terms, we can synthesize these semantic terms into a sentence. For example, "Me See He Left" which can be interpreted to "I saw him in the left direction", "He Quickly Wears a Scarf" and "He Claps" which are self-explanatory. One can easily think of other examples and the grammar can be extended to accommodate more complex sentences.

\begin{tabular}{|c|c|}
\multicolumn{1}{c|}{ TABLE II: GRAMMAR OF MOTION COMMANDS } \\
\hline Rule id & Production rules \\
\hline 1 & $\mathrm{M} \rightarrow \mathrm{S}|\mathrm{VI}| \mathrm{VT}|\mathrm{O}| \mathrm{A}$ \\
\hline 2 & $\mathrm{M} \rightarrow \mathrm{S}$ VI \\
\hline 3 & $\mathrm{M} \rightarrow \mathrm{S}$ VI A | S A VI \\
\hline 4 & $\mathrm{M} \rightarrow \mathrm{S}$ VT O \\
\hline 5 & $\mathrm{M} \rightarrow \mathrm{S}$ VT O A $\mid \mathrm{S}$ A VT O \\
\hline
\end{tabular}

\section{EXPERIMENTAL RESULTS}

The method is tested against the bench mark of 8 classes of $\mathrm{L}_{2} \mathrm{Ms}$ with their corresponding $\mathrm{L}_{1} \mathrm{Ms}$. We conducted the experiments in two ways: one is by using $\mathrm{k}$-fold cross validation where $k=3$ and each motion class $\mathrm{L}_{1}$ Mis provided with 300 positive samples and 1000 negative samples, the other one is tested in real-time manner. Four aspects indicating the method's performance has been evaluated: sensitivity, accuracy, specialty and efficiency. While the aspects of different levels are inter-connected, here we only list the results of $\mathrm{L}_{2}$ Mrecognition. Table III shows the experimental results of both $\mathrm{k}$-fold cross validation and real-time testing, where $\mathrm{CV}$ denotes cross validation and RT denotes real-time. Table IV shows the comparison with other methods in accuracy.

Experiments show that the proposed method has achieved remarkable performance with the average sensitivity reaching $90.6 \%$, average specialty $95.7 \%$ and accuracy 95.4\%; Our approach can handle motions with similar submotions, for instance, $\mathrm{L}_{2} \mathrm{M}$ motion "Me", "Wear a scarf" and "See" all contain the sub-motion of "Lift left hand" which is $\mathrm{L}_{1} \mathrm{M}$, the similar sub-motions can offer probability information of the three motions if handled properly, otherwise, it will be mixed up and result in false recognition.

The test platform's software/hardware configuration is as follows: 64-bit Windows 7 Enterprise, Intel ${ }^{\circledR}$ Core ${ }^{\mathrm{TM}} 2$ Duo CPU E7500 @2.93GHz (Duo) and 4GB RAM. The average time consumption of each $\mathrm{L}_{2} \mathrm{Mis} 6 \mathrm{~ms}$, much faster than the real-time motion data providing, which is at a rate of $50 \mathrm{fps}$ (20ms each frame), thus the efficiency of our approach is 
guaranteed.

TABLE III: EXPERIMENTAL RESULTS

\begin{tabular}{|c|c|c|c|c|c|c|}
\hline \multirow{2}{*}{$\mathrm{L}_{2} \mathrm{M}$} & \multicolumn{2}{|c|}{ Sensitivity $(\%)$} & \multicolumn{2}{|c|}{ Specialty (\%) } & \multicolumn{2}{|c|}{ Accuracy (\%) } \\
\hline & $\mathrm{CV}$ & RT & $\mathrm{CV}$ & $\mathrm{RT}$ & $\mathrm{CV}$ & RTs \\
\hline $\mathrm{Me}$ & 88.4 & 76.1 & 98.7 & 92.4 & 92.8 & 91.3 \\
\hline Claps & 97.6 & 89.4 & 96.6 & 94.7 & 98.4 & 97.6 \\
\hline Wear a Scarf & 100 & 95.8 & 100 & 100 & 100 & 97.8 \\
\hline See & 95.5 & 86.7 & 100 & 97.2 & 100 & 96.8 \\
\hline Stop & 100 & 93.5 & 97.8 & 94.3 & 100 & 94.5 \\
\hline $\begin{array}{c}\text { Stretch } \\
\text { Oneself }\end{array}$ & 100 & 91 & 100 & 93.4 & 100 & 92.3 \\
\hline Left Direction & 100 & 100 & 100 & 100 & 100 & 100 \\
\hline $\begin{array}{c}\text { Right } \\
\text { Direction }\end{array}$ & 100 & 92.6 & 100 & 93.5 & 100 & 93.1 \\
\hline
\end{tabular}

TABLE IV: COMPARISON WITH OTHER METHODS

\begin{tabular}{|c|c|}
\hline Method & Accuracy \\
\hline Vieira et al. $[15]$ & $84.8 \%$ \\
\hline Raptis et al. (only correlation) [5] & $89.9 \%$ \\
\hline Raptis et al. (energy + correlation) [5] & $96.9 \%$ \\
\hline Our approach & $95.4 \%$ \\
\hline
\end{tabular}

\section{CONCLUSION}

In this paper, we proposed a hierarchical method to recognize three different levels of motions and obtained good performance.SVD together with SVM show great power of multi-class classification, HMM and pattern matching are flexible tools to capture and cope with spatiotemporal variations of similar motions. In a high level of motion recognition, grammar-based method can be an appropriate choice. Future works will be focused on finding other good features in motions as well as facilitating training data collection.

\section{ACKNOWLEDGMENT}

We would like to thank members of DALab in School of Software who offer valuable advices and help in implementing part of the recognition framework and data collection.

\section{REFERENCES}

[1] R. M. Baecker and W. Buxton, Human-Computer Interaction: A Multidisciplinary Approach, 1987.

[2] C. Li, P. R. Kulkarni, and B. Prabhakaran, "Segmentation and recognition of motion capture data stream by classification," Multimedia Tools and Applications, vol. 35, no. 1, pp. 55-70, 2007.
[3] L. Kovar and M. Gleicher, "Automated extraction and parameterization of motions in large data sets," ACM Trans. on Graphics (TOG), pp. 559-568, 2004.

[4] J. Alon, V. Athitsos, and S. Sclaroff, "Accurate and efficient gesture spotting via pruning and subgesture reasoning," Computer Vision in Human-Computer Interaction, pp. 189-198, 2005.

[5] M. Raptis, D. Kirovski, and H. Hoppe, "Real-time classification of dance gestures from skeleton animation," in Proc. 2011 ACM SIGGRAPH/Eurographics Symposium on Computer Animation, 2011, pp. 147-156.

[6] H. K. Lee and J. H. Kim, "An HMM-based threshold model approach for gesture recognition," IEEE Trans. on Pattern Analysis and Machine Intelligence, 1999, vol. 21, no. 10, pp. 961-973.

[7] D. Kim, J. Song, and D. Kim, "Simultaneous gesture segmentation and recognition based on forward spotting accumulative HMMs," Pattern Recognition, vol. 40, no. 11, pp. 3012-3026, 2007.

[8] Z. Lin, Z. Jiang, and L. S. Davis, "Recognizing actions by shapemotion prototype trees," in Proc. 2009 IEEE 12th International Conference on Computer Vision, 2009, pp. 444-451.

[9] Q. Gu, J. Peng, and Z. Deng, "Compression of human motion capture data using motion pattern indexing," in Proc. Computer Graphics Forum, 2009, pp. 1-12.

[10] M. Ryoo and J. Aggarwal, "Stochastic representation and recognition of high-level group activities," International Journal of Computer Vision, vol. 93, no. 2, pp. 183-200, 2011.

[11] A. F. Bobick, "Movement, activity and action: The role of knowledge in the perception of motion," Philosophical Trans. of the Royal Society of London. Series B: Biological Sciences, vol. 352, no. 1358, pp. 1257-1265, 1997.

[12] G. H. Golub and C. Reinsch, "Singular value decomposition and least squares solutions," Numerische Mathematik, 1970, vol. 14, no. 5, pp. 403-420.

[13] C. W. Hsu and C. J. Lin, "A comparison of methods for multiclass support vector machines," IEEE Trans. on Neural Networks, 2002, vol. 13 , no. 2 , pp. $415-425$.

[14] M. Muller and T. Roder, "Motion templates for automatic classification and retrieval of motion capture data," in Proc. 2006 ACM SIGGRAPH/Eurographics Symposium on Computer Animation, Vienna, Austria, 2006, pp. 137-146.

[15] A. W. Vieira et al., "Stop: Space-time occupancy patterns for 3dactionrecognition from depth map sequences," CIARP, 2012.

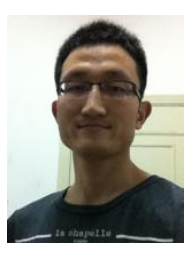

Jianchao Lv was born in Shandong Province on March 30, 1989. He received BS degree in software engineering from Software Engineering Institute of East China Normal University, Shanghai, China. His research interests include human computer interaction and machine learning. He is now a second year graduate student in School of Software, Shanghai Jiaotong University pursing his Master Degree of Engineering.

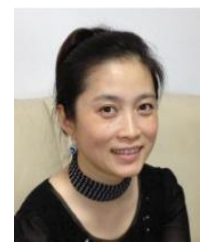

Shuangjiu Xiao was born in Sichuan Province on September 9, 1973. She received Ph.D. degree of Computer Aided Design in 2002 from Northwestern Polytechnical University, Xi'an, China. The major field of study of her includes computer graphics, human computer interaction.

She is an associate professor of School of Software of Shanghai Jiao Tong University. She did Postdoctoral research during 2002-2004 in Computer Science and Technology Department of SJTU. She has about 70 papers published.

Prof. Xiao is a member of the China Graphics Society and the China Computer Federation. 